\title{
Transplacental transfer of dengue
}

\section{D Ekanayake ${ }^{1}$, S Padumadasa ${ }^{2}$, R Premaratna ${ }^{3}$, S Rajendrajith ${ }^{4}$, W W S R M H Samaranayake ${ }^{2}$}

Ceylon Medical Journal 2014; 59: 145-146

\section{Introduction}

Dengue is endemic in Sri Lanka and imposes a great socioeconomic burden. Dengue and dengue hemorrhagic fever (DHF) have a diverse presentation during pregnancy with potential catastrophic consequences as it is difficult to distinguish the critical phase due to anatomical and physiological changes of pregnancy which may mask clinical and haematological features. Furthermore, clinical and laboratory parameters may be attributed to preeclampsia and HELLP syndrome. Uncertainty exists about the possibility and the impact of trans-placental dengue infection mainly due to lack of data. Vertical transmission of dengue, although not widely reported, may carry grave consequences for the neonate $[1,2]$.

\section{Case report}

A 36-years old primigravida, with an uneventful antenatal history presented at 35 weeks of gestation with fever and myalgia of two days duration. She was haemodynamically stable on admission but appeared flushed. She complained of reduced fetal movements for 12 hours and cardiotocography showed marked fetal tachycardia and reduced baseline variability with prolonged deceleration. Full blood count showed a white cell count of $8.9 \times 10^{3} / \mu \mathrm{l}$ with a differential count of neutrophils $76 \%$ and lymphocytes $21 \%$. Platelet count was $56 \times 10^{3} / \mu 1$, haemoglobin concentration was $11.2 \mathrm{~g} / \mathrm{dl}$ and a haematocrit was 34\%. Liver enzymes AST was 408 $\mu / 1$ and ALT was $188 \mu / 1$ respectively. During emergency caesarean section meconium stained liquor was observed but there was no evidence of placental abruption. The baby weighed $1.97 \mathrm{~kg}$ and had Apgar scores of 8, 9, 10 at 1, 5 and 10 minutes respectively. After caesarean section, the mother's thrombocytopenia worsened to $18 \times 10^{3} / \mu 1$ on day two and to $4 \times 10^{3} / \mu$ l on day three. Haemoglobin was $10.6 \mathrm{~g} / \mathrm{dl}$ on day 2 and $10.4 \mathrm{~g} / \mathrm{dl}$ on day 3 . Haematocrit was $34 \%$ on day two and $36 \%$ on day three. There was uterine bleeding on day two and three and was managed with intravenous tranexamic acid and rectal misoprostol.
Thrombocytopenia improved to $8 \times 10^{3} / \mu 1$ on day four, $28 \times 103 / \mu 1$ on day seven and $104 \times 10^{3} / \mu 1$ on day ten. Dengue IgM antibody, was positive on day six of illness. The patient recovered subsequently.

The neonate developed fever of $102^{\circ} \mathrm{F}$ and thrombocytopenia, platelet count was $73 \times 103 / \mu 1$ (day 5) and $7 \times 103 / \mu 1$ (day 11). There was evidence of plasma leakage from day five onwards which worsened despite treatment. A septic screen yielded negative results, C-reactive protein was normal. Blood picture showed macrocytic anaemia with no evidence of bacterial infection. The liver enzymes were markedly elevated from day 11 (AST $1300 \mathrm{i} \mu / \mathrm{l}$, ALT $497 \mathrm{i \mu} / \mathrm{l}$ ). Dengue antibodies, IgM, IgG and NS1 antigen (Platelia Dengue NS1 Ag assay) done on day five were positive. Abdominal ultrasound showed a small amount of pericholecystic fluid. Chest X-ray and ultrasound brain were normal. The neonate succumbed on day 12 .

\section{Discussion}

The initial differential diagnosis in the woman was sepsis or DHF. Presence of flushing of the skin is suggestive of dengue but is known to occur during pregnancy. Although leucopenia with relative lymphocytosis is expected in dengue infection, physiological neutrophil leucocytosis that occurs around parturition causes difficulties in diagnosing viral infections. Haemodilution during pregnancy makes it difficult to interpret haematological parameters in detecting fluid leakage. Although she had significant thrombocytopenia and high liver enzymes, it is not specific to dengue and illnesses such as HELLP syndrome needs to be considered during such circumstances. DHF was diagnosed on day five after detecting dengue antibodies. Earlier diagnosis of the infection could have been done only by dengue PCR or detection of dengue NS1 antigen.

Similarly, the differential diagnosis in the neonate included septicaemia. However, the possibility of DHF due to vertical infection was suspected. Neonatal

${ }^{1}$ Gynaecology and Obstetrics Unit, Colombo North Teaching Hospital, Departments of ${ }^{2}$ Obstetrics and Gynaecology, ${ }^{3}$ Medicine and ${ }^{4}$ Paediatrics, Faculty of Medicine, University of Kelaniya, Ragama, Sri Lanka.

Correspondence: CDE, e-mail: <cdekanayake2000@yahoo.co.uk>. Received 28 July 2014 and revised version accepted 6 October 2014. Competing interests: none declared. 
septicaemia was excluded due to normal CRP levels, negative septic screen and the absence of a marked neutrophil leucocytosis. The neonate was positive for dengue NS1 Ag as well as for dengue IgM confirming acute dengue infection by vertical transmission. Although post delivery dengue infection was a possibility, this was unlikely due to the very short time gap between the delivery and the onset of the illness and the detection of dengue IgM.

\section{References}

1. Thaithumyanon P, Thisyakorn U, Deerojnawong J, Innis B. Dengue infection complicated by severe hemorrhage and vertical transmission in a parturient woman. Clinical Infectious Diseases 1994; 18: 248-9.

2. Fatimil L, Mollah A, Ahmed S, Rahman M. Vertical transmission of dengue: first case report from Bangladesh. Southeast Asian Journal of Tropical Medicine and Public Health 2003; 34: 800-3. 